\title{
Ground-state electromagnetic moments of calcium isotopes
}

\author{
R. F. Garcia Ruiz, ${ }^{1,{ }^{*}}$ M. L. Bissell, ${ }^{1}$ K. Blaum, ${ }^{2}$ N. Frömmgen, ${ }^{3}$ M. Hammen, ${ }^{3}$ J. D. Holtt, ${ }^{4,5,6}$ M. Kowalska, ${ }^{7}$ K. Kreim, ${ }^{2}$ \\ J. Menéndez, ${ }^{4,5,8}$ R. Neugart, ${ }^{2,3}$ G. Neyens, ${ }^{1}$ W. Nörtershäuser, ${ }^{4}$ F. Nowacki, ${ }^{9}$ J. Papuga, ${ }^{1}$ A. Poves, ${ }^{10}$ A. Schwenk, $, 4,5$ \\ J. Simonis, ${ }^{4,5}$ and D. T. Yordanov ${ }^{2}$ \\ ${ }^{1}$ KU Leuven, Instituut voor Kern-en Stralingsfysica, B-3001 Leuven, Belgium \\ ${ }^{2}$ Max-Plank-Institut für Kernphysik, D-69117 Heidelberg, Germany \\ ${ }^{3}$ Institut für Kernchemie, Universität Mainz, D-55128 Mainz, Germany \\ ${ }^{4}$ Institut für Kernphysik, Technische Universität Darmstadt, D-64289 Darmstadt, Germany \\ ${ }^{5}$ Extreme Matter Institute EMMI, GSI Helmholtzzentrum für Schwerionenforschung GmbH, D-64291 Darmstadt, Germany \\ ${ }^{6}$ TRIUMF, 4004 Wesbrook Mall, Vancouver, British Columbia, V6T 2A3, Canada \\ ${ }^{7}$ CERN, European Organization for Nuclear Research, Physics Department, CH-1211 Geneva 23, Switzerland \\ ${ }^{8}$ Department of Physics, University of Tokyo, Hongo, Tokyo 113-0033, Japan \\ ${ }^{9}$ IPHC, IN2P3-CNRS and Universite Louis Pasteur, F-67037 Strasbourg, France \\ ${ }^{10}$ Departamento de Física Teórica and IFT-UAM/CSIC, Universidad Autónoma de Madrid, E-28049 Madrid, Spain
}

(Received 10 November 2014; revised manuscript received 8 April 2015; published 30 April 2015)

\begin{abstract}
Background: The neutron-rich calcium isotopes have gained particular interest as evidence of closed-shell structures has recently been found in two exotic nuclei, at $N=32$ and $N=34$. Additionally, the study of such neutron-rich systems has revealed new aspects of nuclear forces, in particular regarding the role of three-nucleon forces.

Purpose: We study the electromagnetic properties of Ca isotopes around the neutron number $N=32$.

Methods: High-resolution bunched-beam collinear laser spectroscopy was used to measure the optical hyperfine spectra of the ${ }^{43-51} \mathrm{Ca}$ isotopes.

Results: The ground-state magnetic moments of ${ }^{49,51} \mathrm{Ca}$ and quadrupole moments of ${ }^{47,49,51} \mathrm{Ca}$ were measured for the first time, and the ${ }^{51} \mathrm{Ca}$ ground-state spin $I=3 / 2$ was determined in a model-independent way. Our experimental results are compared with state-of-the-art shell-model calculations using both phenomenological interactions and microscopic interactions derived from chiral effective field theory.

Conclusions: The results for the ground-state moments of neutron-rich isotopes are in excellent agreement with predictions of interactions derived from chiral effective field theory including three-nucleon forces. Lighter isotopes illustrate the presence of particle-hole excitations of the ${ }^{40} \mathrm{Ca}$ core in their ground state. Our results provide a critical test of modern nuclear theories, and give direct answer to the evolution of ground-state electromagnetic properties in the Ca isotopic chain across three doubly closed-shell configurations at $N=20$, 28,32 of this unique system.
\end{abstract}

DOI: 10.1103/PhysRevC.91.041304

The existence of doubly magic nuclei has played a key role in our understanding of nuclear structure. Doubly magic nuclei have been the basis to develop the shell model, and are an ideal probe to test our knowledge of nuclear interactions by comparing experimental data with shell-model predictions [1,2]. Such shell-model calculations depend on the effective Hamiltonian used, a suitable valence space to capture the low-energy degrees of freedom, and consistent effective operators. Although effective charges and $g$ factors are widely used in shell-model calculations, they are not completely understood. Furthermore, their orbital [3] and valence-space [4] dependence and connection to two-body currents (meson-exchange currents), known to be important for magnetic moments in light nuclei [5], are under discussion.

*ronald.fernando.garcia.ruiz@cern.ch

Published by the American Physical Society under the terms of the Creative Commons Attribution 3.0 License. Further distribution of this work must maintain attribution to the author(s) and the published article's title, journal citation, and DOI.
PACS number(s): 21.10.Ky, 21.45.Ff, 21.60.Cs, 42.62.Fi

Having a closed proton shell, $Z=20$, and two naturally occurring doubly magic isotopes, ${ }^{40} \mathrm{Ca}$ and ${ }^{48} \mathrm{Ca}$, the calcium isotopic chain has always been considered a prime benchmark for nuclear structure, both from a theoretical [6] and an experimental perspective [7]. Recently, special attention has turned to the evolution of the structure beyond $N=28$, where additional shell closures have been suggested at $N=$ 32 [8] and $N=34$ [9]. These neutron-rich calcium isotopes have also gained exceptional interest from the theoretical side [10-14], as their properties reveal new aspects of nuclear forces, in particular regarding the role of three-nucleon $(3 \mathrm{~N})$ forces [10,11] (for a review on $3 N$ forces see [15]).

Spectroscopic properties in the $\mathrm{Ca}$ region are well described by phenomenological shell-model interactions, such as KB3G [16] and GXPF1A [17]. These interactions start from a ${ }^{40} \mathrm{Ca}$ core and two-nucleon $(N N)$ forces and refit part of the interactions to experimental data in the $p f$ shell, to compensate for neglected many-body effects (both due to $3 N$ and many-body correlations) [2]. Normal and superdeformed bands in ${ }^{40} \mathrm{Ca}$ have been understood as due to particle-hole excitations of protons and neutrons from the $s d$ shell into the $p f$ orbits, and have been well described 
using the SDPF.SM shell model interaction starting from a virtual ${ }^{28} \mathrm{Si}$ core [18]. These calculations showed that the ${ }^{40} \mathrm{Ca}$ ground state is very correlated. In the last years, valence-shell interactions have been derived from $N N$ and $3 N$ forces based on chiral effective field theory [10], fitted only to few-nucleon systems. Investigating the reliability of these microscopic $N N+3 N$ interactions is a matter of general interest as they have direct implications for the modeling of astrophysical systems [19]. The $N N+3 N$ interactions provide a good description of the shell structure and the spectra of neutron rich calcium isotopes in an extended valence space $\left(f_{p g_{9 / 2}}\right)$ [14]. The electric quadrupole $(E 2)$ transitions obtained from both phenomenological and microscopic interactions exhibit good agreement using the neutron effective charge: $e_{n}=0.5 e$. On the other hand, phenomenological interactions and $N N+3 N$ disagree in effective nucleon $g$ factors needed to reproduce the magnetic (M1) transition strengths [14].

Despite the remarkable differences, both phenomenological and microscopic $N N+3 N$ interactions give a similar description of neutron separation (binding) energies and lowlying excitation energies of $\mathrm{Ca}$ isotopes from $N=22$ up to $N=32[8,9,20]$. As illustrated in [21], such observables might however be insensitive to cross-shell correlations. Therefore, there is a need to measure additional observables like electromagnetic moments, which further test the above models and might provide a deeper insight to developing improved shell-model interactions.

Magnetic moments and $g$ factors, $g=\mu /\left(I \mu_{N}\right)$, of isotopes near shell closures are very sensitive to the occupancy of particular orbitals by valence particles (or holes). The quadrupole moments on the other hand are directly sensitive to nuclear shell structure [22]. While the terms closed shell or magic number may lack a rigid definition, the electromagnetic moments provide a more direct probe of the structure involved including cross-shell effects [23,24].

In this Rapid Communication, we report the first measurements of the quadrupole moment of the closed shell -1 isotope ${ }^{47} \mathrm{Ca}$, and the quadrupole and magnetic moment of the closed-shell +1 isotope ${ }^{49} \mathrm{Ca} .{ }^{1}$ Also the magnetic and quadrupole moments of ${ }^{51} \mathrm{Ca}$, having a single-hole with respect to the new $N=32$ subshell closure, are presented, as well as its ground state (g.s.) spin. The experimental data are compared to shell-model calculations using phenomenological interactions, and to calculations including $3 N$ forces based on chiral effective field theory.

At ISOLDE, CERN, exotic Ca isotopes were produced from nuclear reactions induced by a high-energy proton beam ( $1.4 \mathrm{GeV}$; pulses of $2 \mu \mathrm{C}$ typically every $2.4 \mathrm{~s}$ ) impinging on a uranium carbide target. High selectivity for the $\mathrm{Ca}$ reaction products was accomplished by laser ionization [25]. Ions were extracted from the ion source and accelerated up to $30 \mathrm{keV}$ or $40 \mathrm{keV}$ to be mass separated, after which they were injected into the ISOLDE radiofrequency quadrupole (RFQ) beam cooler, ISCOOL [26]. Ions were trapped for

\footnotetext{
${ }^{1} \mathrm{~A}$ previous value for the magnetic moment of ${ }^{49} \mathrm{Ca}$ was suggested from a partial measurement of its hfs and assuming a value for its unknown isotope shift [52].
}

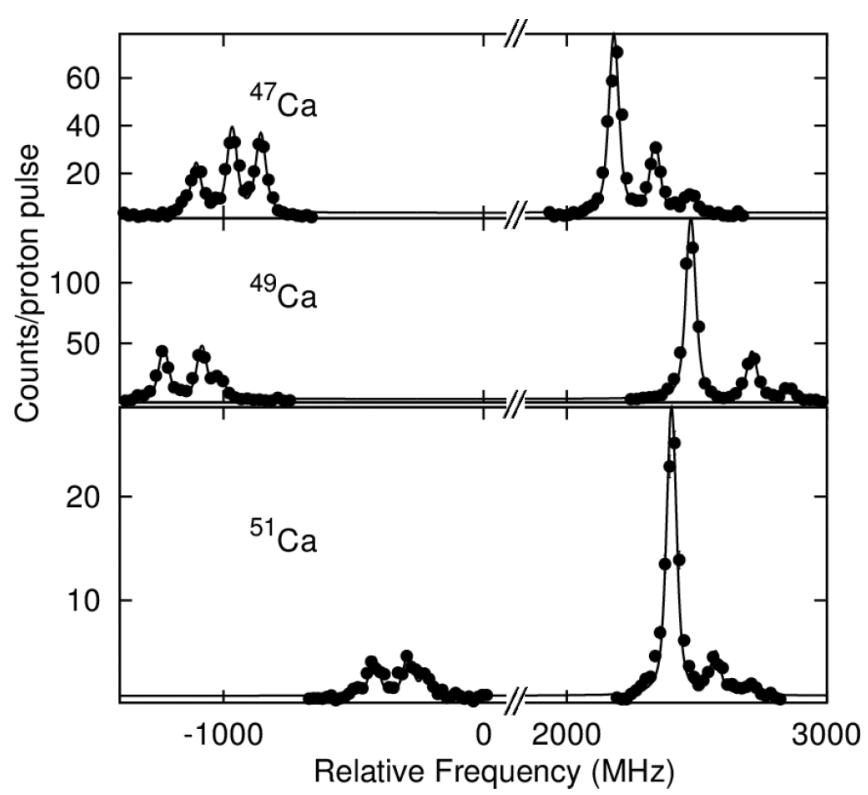

FIG. 1. Examples of hfs spectra measured for the Ca isotopes in the $393 \mathrm{~nm} 4 s^{2} S_{1 / 2} \rightarrow 4 p^{2} P_{3 / 2}$ ionic transition. The lines show the fit with a Voigt profile. Frequency values are relative to the centroid of ${ }^{43} \mathrm{Ca}$.

approximately $50 \mathrm{~ms}$, and extracted bunches of $5 \mu$ s temporal width were distributed to a dedicated beam line for collinear laser spectroscopy experiments (COLLAPS). At COLLAPS, the ion beam was superimposed with a continuous wave (CW) laser beam from a frequency-doubled Ti:Sa laser, providing a 393-nm laser wavelength to excite the $4 s^{2} S_{1 / 2} \rightarrow$ $4 p^{2} P_{3 / 2}$ transition in $\mathrm{Ca}^{+}$. The laser frequency was locked to a Fabry-Perrot interferometer, which was in turn locked to a polarization-stabilized $\mathrm{HeNe}$ laser, reducing the laser frequency drift to $<10 \mathrm{MHz}$ per day.

By changing the ion velocity, and thereby Doppler tuning the laser frequency in the ion rest frame, hyperfine structure (hfs) components could be scanned. Fluorescence photons were detected by a set of four photomultipliers (PMT) at the end of the beam line (see Refs. [27,28] for details). By only accepting signals from the PMT while the ion bunch passed in front of them, background from scattered laser light and PMT dark counts was reduced by a factor of $\sim 10^{4}$. Sample hfs spectra measured during the experiment are shown in Fig. 1. The magnetic hfs constants, $A\left({ }^{2} S_{1 / 2}\right), A\left({ }^{2} P_{3 / 2}\right)$, and quadrupole hfs constants, $B\left({ }^{2} P_{3 / 2}\right)$, were extracted from the fit of Voigt profiles to the experimental spectra by using a $\chi^{2}$-minimization technique as explained, e.g., in Ref. [29]. The values are listed in Table I. Only ${ }^{43} \mathrm{Ca}$ has been studied before in this ionic transition, and our values are in agreement within 1.1 standard deviations. For ${ }^{41} \mathrm{Ca}$ and ${ }^{45} \mathrm{Ca}$, two measurements of the quadrupole hfs constants have been reported in the atomic level system, both relative to that of ${ }^{43} \mathrm{Ca}[30,31]$, yielding the ratios $B\left({ }^{41} \mathrm{Ca}\right) / B\left({ }^{43} \mathrm{Ca}\right)=1.63(1)$, and $B\left({ }^{45} \mathrm{Ca}\right) / B\left({ }^{43} \mathrm{Ca}\right)=-0.94(27)$. The $B$-factor ratio equals the ratio of the quadrupole moments. Thus we can compare the ratio of our $B$ values, measured in the ionic system 
TABLE I. Hyperfine structure values obtained from the fit to the experimental data compared to previous measurements.

\begin{tabular}{lccccc}
\hline \hline$A$ & $I^{\pi}$ & $\begin{array}{c}A\left({ }^{2} S_{1 / 2}\right) \\
(\mathrm{MHz})\end{array}$ & $\begin{array}{c}A\left({ }^{2} P_{3 / 2}\right) \\
(\mathrm{MHz})\end{array}$ & $\begin{array}{c}B\left({ }^{2} P_{3 / 2}\right) \\
(\mathrm{MHz})\end{array}$ & Ref. \\
\hline 43 & $7 / 2^{-}$ & $-806.87(42)$ & $-31.10(30)$ & $-4.2(1.3)$ & \\
& & $-806.40207160(8)$ & & & {$[32]$} \\
& & $-805(2)$ & $-31.9(2)$ & $-6.7(1.4)$ & {$[33]$} \\
& & & $-31.0(2)$ & $-6.9(1.7)$ & {$[34]$} \\
45 & $7 / 2^{-}$ & $-811.99(44)$ & $-31.43(19)$ & $3.1(1.0)$ & \\
47 & $7 / 2^{-}$ & $-860.96(28)$ & $-33.33(13)$ & $12.68(96)$ & \\
49 & $3 / 2^{-}$ & $-1971.02(30)$ & $-75.98(11)$ & $-5.53(40)$ & \\
51 & $3 / 2^{-}$ & $-1499.22(94)$ & $-58.15(54)$ & $5.4(1.8)$ & \\
\hline \hline
\end{tabular}

$B\left({ }^{45} \mathrm{Ca}\right) / B\left({ }^{43} \mathrm{Ca}\right)=-0.74(31)$, to the latter value. They are in agreement within the error bars.

The nuclear spin, $I$, is required to calculate each peak position in the minimization procedure. Since a different set of hfs constants is found for a given spin, the ratio, $R=A\left({ }^{2} P_{3 / 2}\right) / A\left({ }^{2} S_{1 / 2}\right)$, can be used to determine the correct spin for each isotope, as this ratio should be a constant over the entire isotopic chain (neglecting a possible small hyperfine anomaly). As can be seen from Fig. 2, the ratio $R$ remains constant along the $\mathrm{Ca}$ isotopes up to ${ }^{49} \mathrm{Ca}$, using the earlier determined g.s. spins in the fitting procedure. For ${ }^{51} \mathrm{Ca}$ we assumed three possible spins for its ground state and only when $I=3 / 2$ is used, the ratio of the fitted hfs parameters is consistent with those from the other isotopes. Thus $I=$ $3 / 2$ is the g.s. spin of ${ }^{51} \mathrm{Ca}$, confirming earlier tentative assignments [35,36], and in agreement with expectations from the shell model.

Magnetic moments were extracted from the lower state magnetic hfs constants, $A=\mu_{I} B_{0} /(I J)$, where $B_{0}$ is the magnetic field produced by the electrons at the nucleus, and $J$ is the electronic total angular momentum. Since high-precision

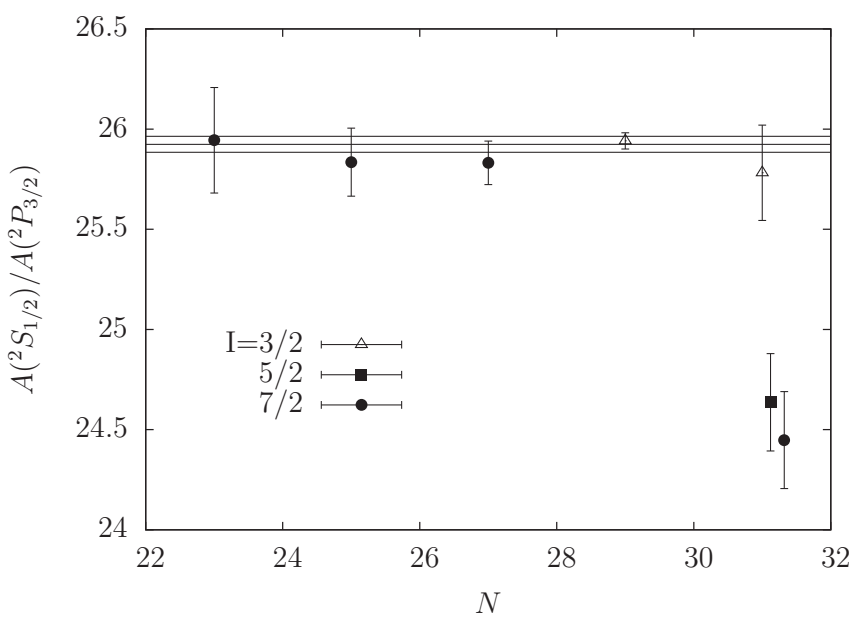

FIG. 2. Ratio between the hfs constants $A\left({ }^{2} P_{1 / 2}\right)$ and $A\left({ }^{2} P_{3 / 2}\right)$. The continuous line shows the average value $A\left({ }^{2} S_{1 / 2}\right) / A\left({ }^{2} P_{3 / 2}\right)=$ 25.92(3). Hyperfine structure spectra of ${ }^{51} \mathrm{Ca}$ were fitted assuming different g.s. spin values of $I=3 / 2,5 / 2,7 / 2$. values of $A\left({ }^{2} S_{1 / 2}\right)=-806.40207160(8) \mathrm{MHz}$ [32] and $\mu=$ $-1.3173(6)$ [37] are known for ${ }^{43} \mathrm{Ca}$, this isotope was used as a reference to calculate the other magnetic moments from the measured $A$ values. The results are shown in Table II, where we compare our data to earlier reported values for ${ }^{45} \mathrm{Ca}$ and ${ }^{47} \mathrm{Ca}$.

Quadrupole moments, $Q$, were obtained from the quadrupole hfs constant, $B=e Q V_{J J}$, with $e$ the electron charge, and $V_{J J}$ the electric field gradient (EFG) produced by the electrons at the nucleus, the latter being isotope independent. To extract quadrupole moments from the measured hfs $B$ parameters, a calculated value for the EFG, $e V_{J J}=$ 151.3(7) $\mathrm{MHz} / \mathrm{b}$, was taken from atomic-physics calculations based on relativistic coupled-cluster theory (RCC) [40]. Independent values calculated from many-body perturbation theory (MBPT) $[43,44]$ agree with the value from RCC within $3 \%$. The extracted quadrupole moments are shown in Table II. The deviation of our value for ${ }^{43} \mathrm{Ca}$ from the literature values is attributed to the low statistics of our data for this isotope combined with a poorly resolved hyperfine splitting in the excited state. Note however that our ratio of the ${ }^{45} \mathrm{Ca}$ to ${ }^{43} \mathrm{Ca}$ quadrupole moment is consistent with the value measured in the atomic system.

Since the $g$ factors are sensitive to the valence-particle configuration, it is illustrative to study their evolution along the $\mathrm{Ca}$ isotopic chain. The horizontal lines in Fig. 3 show the effective single-particle values $\left(g_{\text {eff }}^{\nu}=0.8 g_{\text {free }}^{\nu}\right)$ for the different shell-model orbits. The isotope ${ }^{39} \mathrm{Ca}(N=19)$ has a $g$ factor close to the $d_{3 / 2}$ effective single-particle value, confirming the hole nature of this isotope. Once the $d_{3 / 2}$ orbit is filled, the fairly constant $g$-factor values from $N=21$ up to $N=27$ are in agreement with that of an odd neutron in the $f_{7 / 2}$ orbital.

As expected, the measured $g$ factor of ${ }^{49} \mathrm{Ca}$ is close to the effective single-particle value of the $p_{3 / 2}$ orbit, and a similar value would be expected for ${ }^{51} \mathrm{Ca}$. However, a deviation from this value is observed, indicating an appreciable contribution from the mixing with configurations due to neutron excitations across $N=32$, which seems to contradict the closed-shell nature of $N=32$. The isotope ${ }^{51} \mathrm{Ca}$ is an exceptional case for testing different shell-model interactions as excitations across $N=32$ can be of $M 1$-type (from $p_{3 / 2}$ into $p_{1 / 2}$ ) and therefore even a one percent mixing of those configurations in the wave function is sufficient to induce a $\sim 20 \%$ change of the $g$ factor [46].

The measured and calculated magnetic moments of the $\mathrm{Ca}$ isotopes are shown in the upper panel of Fig. 4. A ${ }^{40} \mathrm{Ca}$ core is assumed in the calculations with the GXPF1A and $\mathrm{KB} 3 \mathrm{G}$ phenomenological interactions, as well as for the calculations with the microscopic $N N+3 N$ interaction. To investigate the effect of breaking the ${ }^{40} \mathrm{Ca}$ core we also compare to a large-scale shell model calculation using the phenomenological interaction SDPF.SM starting from a virtual ${ }^{28} \mathrm{Si}$ core. For the KB3G and GXPF1A interactions, neutrons were allowed to occupy the $p f$ shell, while an extended valence space including the $0 g_{9 / 2}$ orbital ( $p f g_{9 / 2}$ space) was used for the $N N+3 N$ calculations. Excitations of neutrons and protons from the upper $s d$ shell into the $p f$ shell are allowed with the SDPF.SM interaction. Bare spin and orbital 
TABLE II. Quadrupole and magnetic moments obtained from the measured hfs constants (Table I). The magnetic moments were obtained using the reference isotope ${ }^{43} \mathrm{Ca}$, with $A\left({ }^{2} P_{3 / 2}\right)=-806.40207160(8) \mathrm{MHz}$ [32]. Quadrupole moments were extracted using the calculated electric field gradient, $e V_{J J}=151.3(7) \mathrm{MHz} / \mathrm{b}$ [40]. Data are compared to calculations using the $N N+3 N$ interaction.

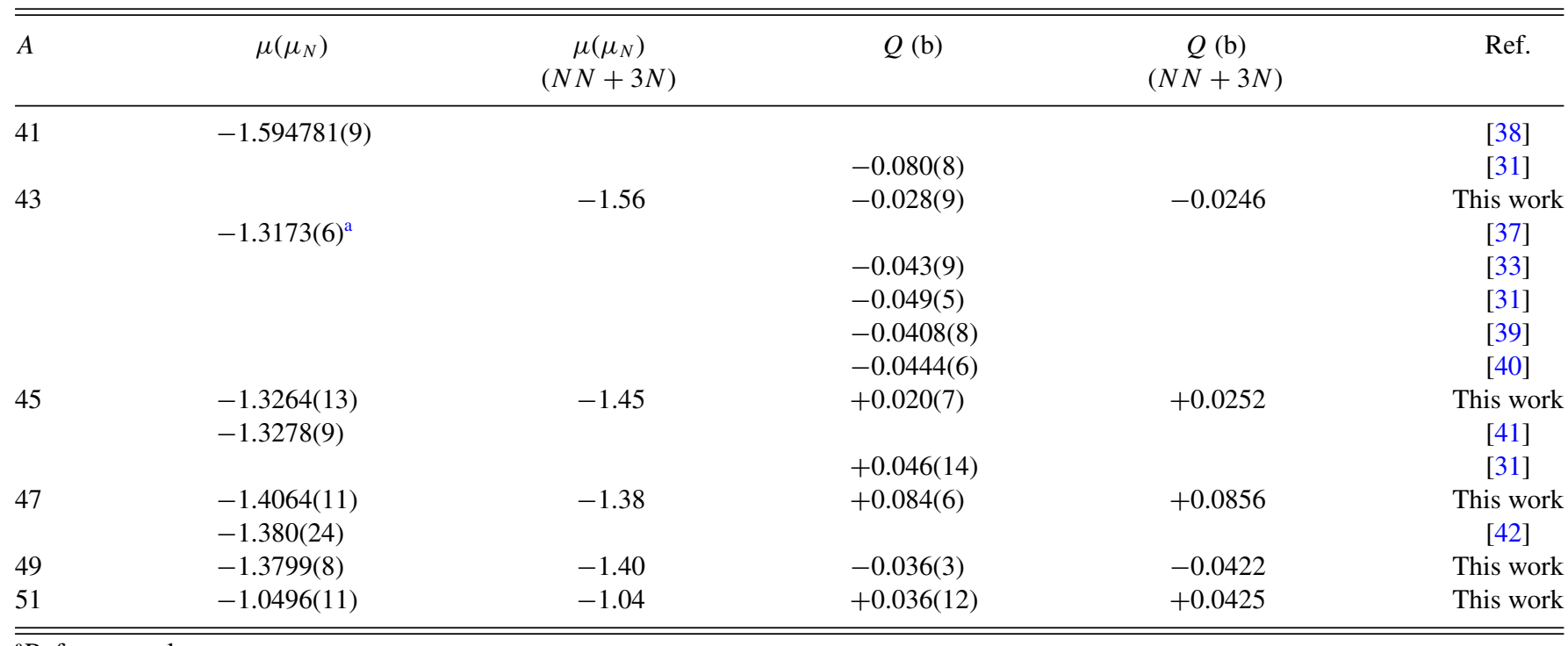

${ }^{\text {a }}$ Reference value.

$g$ factors were used in all theories to calculate the magnetic moments.

The disagreement between the shell-model calculations starting from a ${ }^{40} \mathrm{Ca}$ core and the experimental magnetic moments of ${ }^{41,43,45} \mathrm{Ca}$ suggests that nucleon excitations across the $s d$ shell are important in the vicinity of $N=20$. Indeed, large-scale shell model calculations using the SDPF.SM interaction are closer to the experimental values. These calculations include up to $6 \mathrm{p}-6 \mathrm{~h}$ for ${ }^{41} \mathrm{Ca}, 4 \mathrm{p}-4 \mathrm{p}$ for ${ }^{43} \mathrm{Ca}$, and $2 \mathrm{p}-2 \mathrm{p}$ for the other isotopes. A similar conclusion on the importance of cross-shell correlation across $N=20$ was obtained from experimental $g\left(2^{+}\right)$factors and $B(E 2)$ values of ${ }^{42,44} \mathrm{Ca}[47,48]$ as well as the calcium isotope shifts [49]. For the heavier $\mathrm{Ca}$ isotopes, all theoretical calculations describe

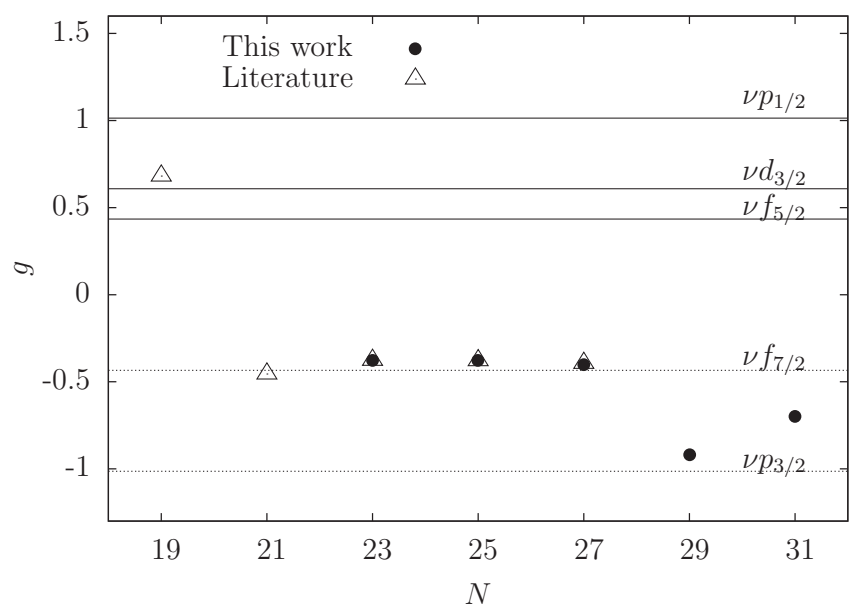

FIG. 3. Measured $g$ factors compared with literature values and effective single-particle values (lines) using $g_{s}^{v}=-3.041$ and $g_{l}^{v}=$ $0.0\left(g_{\text {eff }}^{v}=0.8 g_{\text {free }}^{v}\right)$. The magnetic moment of ${ }^{39} \mathrm{Ca}$ was taken from Ref. [45]. The experimental error bars are smaller than the symbols. the experimental value rather well (see Fig. 4), indicating that from $N=27$ and beyond, the assumption of a rigid ${ }^{40} \mathrm{Ca}$ core works well. Especially, the calculations with the $N N+3 N$ interaction give a very good agreement for ${ }^{47,49,51} \mathrm{Ca}$. Considering that from the measured $g$ factor a mixed ground state wave function is expected (Fig. 3), the excellent agreement for the microscopic calculations, which are not fitted to this mass region, is remarkable. The fact that the calculated values for the phenomenological KB3G and GXPF1A lay on opposite side of the experimental value is due to the different contributions of $\left(p_{1 / 2}\right)^{2}\left(p_{3 / 2}\right)^{1}$ and $\left(p_{1 / 2}\right)^{1}\left(p_{3 / 2}\right)^{2}$ configurations. Certainly, the magnetic moment is highly sensitive to matrix elements involving the $p_{3 / 2}-p_{1 / 2}$ spin-orbit partners. The ratio of $\left(p_{1 / 2}\right)^{1}\left(p_{3 / 2}\right)^{2}$ to $\left(p_{3 / 2}\right)^{3}$ configurations in ${ }^{51} \mathrm{Ca}$ is a measure for these cross-shell excitations across $N=32$ : it is almost twice larger with $N N+3 N$ and GXPF1A (3.5\% and $4.0 \%$, respectively) than in $\mathrm{KB} 3 \mathrm{G}(2.0 \%)$. Larger cross-shell excitations reduce the absolute value of the magnetic moment. On the other hand, due to stronger pairing, the $N N+3 N$ and $\mathrm{KB} 3 \mathrm{G}$ interactions have a two times larger ratio of $\left(p_{1 / 2}\right)^{2}\left(p_{3 / 2}\right)^{1}$ over $\left(p_{1 / 2}\right)^{1}\left(p_{3 / 2}\right)^{2}$ configurations than GXPF1A, 1.6 and 1.9 compared to 0.9 . These cross-shell excitations increase the absolute value of the magnetic moment.

In the lower panel of Fig. 4, the experimental and calculated quadrupole moments are shown. The theoretical results assume neutron and proton effective charges, $e_{n}=0.5 e$ and $e_{p}=$ $1.5 e$, respectively (protons in the valence space are allowed for the SDPF.SM interaction only). All interactions exhibit in general a good description of the experimental quadrupole moments. The only deviation exists for $N=27$, where $\mathrm{KB} 3 \mathrm{G}$ and GXPF1A slightly disagree with the experimental value, while $N N+3 N$ and SDPF.SM agrees nicely. The agreement between calculated and experimental values also confirms the values of effective charges used around $N=20[18,49]$, and more recently around $N=28$ [50]. Earlier studies of $N \sim Z$ 


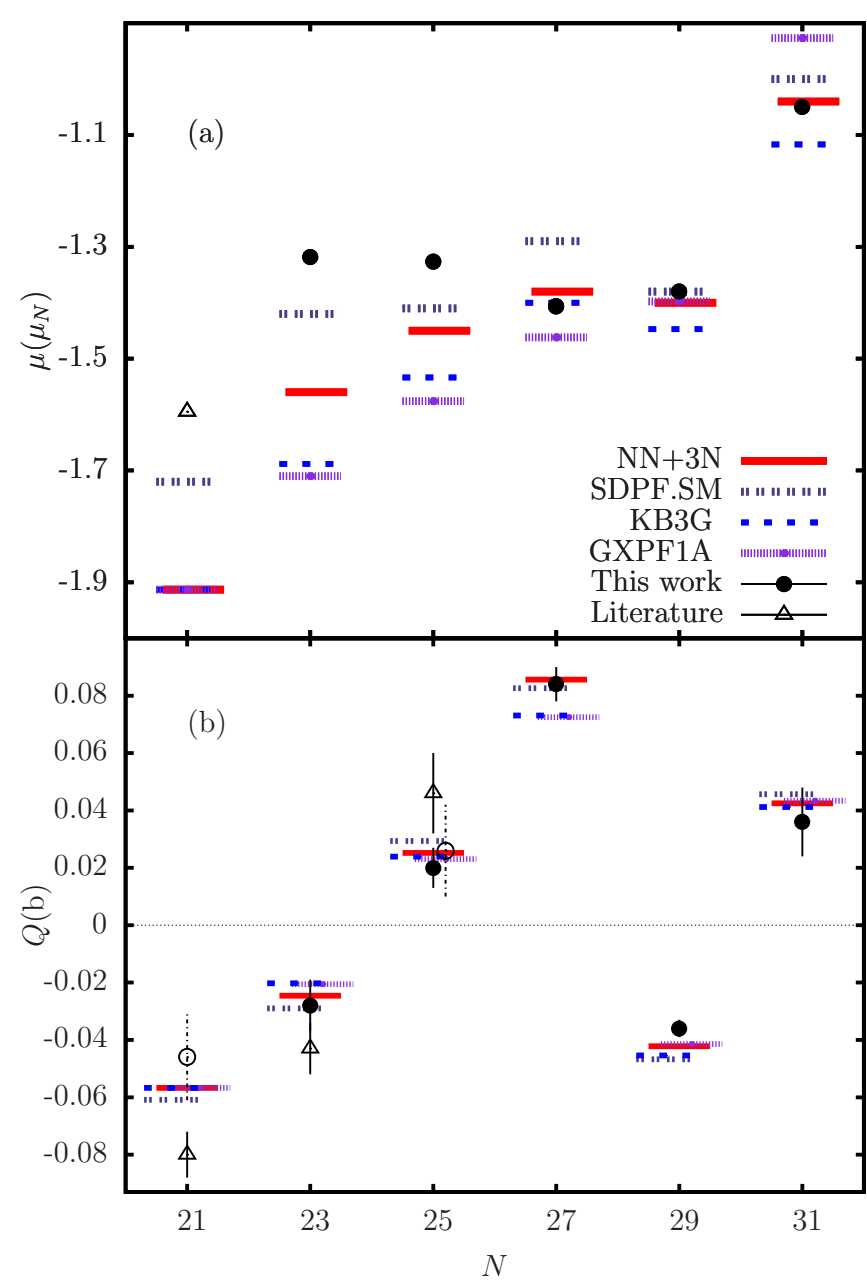

FIG. 4. (Color online) Measured magnetic and quadrupole moments of $\mathrm{Ca}$ isotopes. Results are compared with theoretical predictions from phenomenological interactions (KB3G, GXPF1A, SDPF.SM) and calculations including three-nucleon forces $(N N+$ $3 N$ ). Experimental literature values (empty triangles) are given in Table II. The open circles show the values calculated from the ratios $B\left({ }^{41} \mathrm{Ca}\right) / B\left({ }^{43} \mathrm{Ca}\right)=1.63(1), \quad B\left({ }^{45} \mathrm{Ca}\right) / B\left({ }^{43} \mathrm{Ca}\right)=-0.94(27) \quad[31]$ and relative to our value of $Q\left({ }^{43} \mathrm{Ca}\right)$.

isotopes, where the $f_{7 / 2}$ orbital is dominant, suggested values of $e_{n}=0.8 e$ and $e_{p}=1.15 e$ [51], opening a discussion on the possible orbital dependence of the effective charges in the $p f$ shell [3].
In summary, bunched-beam collinear laser spectroscopy was used to measure the hfs spectra in the Ca II resonance transition from ${ }^{43-51} \mathrm{Ca}$. Our results allowed a direct g.s. spin determination for ${ }^{51} \mathrm{Ca}$. The quadrupole moments of ${ }^{47,49,51} \mathrm{Ca}$, and magnetic moments of ${ }^{49,51} \mathrm{Ca}$ were measured for the first time. We compared these results with new shell-model calculations using a microscopic interaction derived from chiral effective field theory, including $N N+3 N$ forces, and fitted only to isotopes up to mass $A=4$. Comparison was also made with existing and new calculations using phenomenological interactions. Large discrepancies among the measured magnetic moments and the calculated values using a ${ }^{40} \mathrm{Ca}$ core were observed around $N=20$. Large-scale shell model calculations in the $s d-p f$ valence space are required to reproduce better the observed magnetic moments. Further developments of microscopic interactions in the complete $s d$ - $p f$ valence space for both protons and neutrons are needed to provide a consistent description for all observables of Ca isotopes from below $N=20$ up to above $N=32$. For $N \geqslant 27$, the calculations with $N N+3 N$ forces derived from chiral effective field theory provide excellent agreement for both the magnetic and quadrupole moments. Through the gradual filling of the $f_{7 / 2}$ and $p_{3 / 2}$ orbits, our results provide a comprehensive study of the basic ingredients employed in shell-model calculations over a wide range of neutrons. At the level of precision of our experimental results, the g.s. quadrupole moments do not reveal any orbital dependence of the effective charges. The larger difference in calculated magnetic moments compared to electric quadrupole moments highlights the need of improved theoretical calculations, e.g., including two-body currents from chiral effective field theory, that compared to the present measurements may provide new insights on the magnetic operator and effective $g$ factors.

This work was supported by the IAP-project P7/12, the GOA grant 15/010 KU Leuven, NSF Grant No. PHY-1068217, the BMBF Contracts No. 05P12RDCIC and No. 06DA70471, the Max-Planck Society, the EU FP7 via ENSAR No. 262010, the DFG through Grant No. SFB 634, ERC Grant No. 307986 STRONGINT, the Helmholtz Alliance HA216/EMMI, and MINECO (SPAIN) (FPA2011-29854). Computations were performed on JUROPA at the Jülich Supercomputing Center. We would like to thank the ISOLDE technical group for their support and assistance. International Research Fellowship of the Japan Society for the Promotion of Science (JSPS) and JSPS KAKENHI Grant No. 26.04323.
[1] B. A. Brown, Prog. Part. Nucl. Phys. 47, 517 (2001).

[2] E. Caurier et al., Rev. Mod. Phys. 77, 427 (2005).

[3] J. J. Valiente-Dobon et al., Phys. Rev. Lett. 102, 242502 (2009).

[4] I. Stetcu and J. Rotureau, Prog. Part. Nucl. Phys. 69, 182 (2013).

[5] S. Pastore, S. C. Pieper, R. Schiavilla, and R. B. Wiringa, Phys. Rev. C 87, 035503 (2013).

[6] I. Talmi, Rev. Mod. Phys. 34, 704 (1962).

[7] W. H. King, Isotope Shifts in Atomic Spectra, 1st ed. (Plenum Press, New York/London, 1984).

[8] F. Wienholtz et al., Nature 498, 346 (2013).

[9] D. Steppenbeck et al., Nature 502, 207 (2013).
[10] J. D. Holt et al., J. Phys. G 39, 085111 (2012).

[11] G. Hagen, M. Hjorth-Jensen, G. R. Jansen, R. Machleidt, and T. Papenbrock, Phys. Rev. Lett. 109, 032502 (2012).

[12] R. Roth et al., Phys. Rev. Lett. 109, 052501 (2012).

[13] V. Somà, A. Cipollone, C. Barbieri, P. Navrátil, and T. Duguet, Phys. Rev. C 89, 061301(R) (2014).

[14] J. D. Holt, J. Menéndez, J. Simonis, and A. Schwenk, Phys. Rev. C 90, 024312 (2014).

[15] H.-W. Hammer, A. Nogga, and A. Schwenk, Rev. Mod. Phys. 85, 197 (2013).

[16] A. Poves et al., Nucl. Phys. A 694, 157 (2001). 
[17] M. Honma, T. Otsuka, B. A. Brown, and T. Mizusaki, Phys. Rev. C 69, 034335 (2004).

[18] E. Caurier, J. Menéndez, F. Nowacki, and A. Poves, Phys. Rev. C 75, 054317 (2007).

[19] K. Helbert et al., Astrophys. J. 773, 11 (2013).

[20] A. T. Gallant et al., Phys. Rev. Lett. 109, 032506 (2012).

[21] P. Isacker and I. Talmi, J. Phys. Conf. Ser. 267, 012029 (2011).

[22] C. H. Townes, H. M. Foley, and W. Low, Phys. Rev. 76, 1415 (1949).

[23] R. J. Blin-Stoyle, Rev. Mod. Phys. 28, 75 (1956).

[24] G. Neyens, Rep. Prog. Phys. 66, 633 (2003).

[25] V. N. Fedosseev et al., Rev. Sci. Instrum. 83, 02 A903 (2012).

[26] E. Mané et al., Eur. Phys. J. A 42, 503 (2009).

[27] J. Papuga et al., Phys. Rev. Lett. 110, 172503 (2013).

[28] K. Kreim et al., Phys. Lett. B 731, 97 (2014).

[29] M. Kowalska et al., Phys. Rev. C 77, 034307 (2008).

[30] E. Bergmann et al., Z. Phys. A 294, 319 (1980).

[31] M. Arnold et al., Z. Phys. A 314, 303 (1983).

[32] F. Arbes et al., Z. Phys. D 31, 27 (1994).

[33] R. E. Silverans et al., Z. Phys. D 18, 351 (1991).

[34] W. Nörtershauser et al., Eur. Phys. J. D 2, 33 (1998).

[35] F. Perrot et al., Phys. Rev. C 74, 014313 (2006).

[36] B. Fornal et al., Phys. Rev. C 77, 014304 (2008).
[37] L. Olschewski, Z. Phys. 249, 205 (1972).

[38] E. Brun et al., Phys. Rev. Lett. 9, 166 (1962).

[39] D. Sundholm and J. Olsen, J. Chem. Phys. 98, 7152 (1993).

[40] B. K. Sahoo, Phys. Rev. A 80, 012515 (2009).

[41] M. Arnold et al., Hyperfine Interact. 9, 159 (1981).

[42] A. Andl et al., Phys. Rev. C 26, 2194 (1982).

[43] K. Z. Yu, L. J. Wu, B. C. Gou, and T. Y. Shi, Phys. Rev. A 70, 012506 (2004).

[44] A.-M. Mårtensson-Pendrill and S. Salomonson, Phys. Rev. A 30, 712 (1984).

[45] T. Minamisono et al., Phys. Lett. B 61, 155 (1976).

[46] R. J. Blin-Stoyle and M. A. Perks, Proc. Phys. Soc. A 67, 885 (1954).

[47] S. Schielke et al., Phys. Lett. B 571, 29 (2003).

[48] M. J. Taylor et al., Phys. Lett. B 605, 265 (2005).

[49] E. Caurier et al., Phys. Lett. B 522, 240 (2001).

[50] L. A. Riley et al., Phys. Rev. C 90, 011305(R) (2014).

[51] R. du Rietz et al., Phys. Rev. Lett. 93, 222501 (2004).

[52] L. Vermeeren et al., Proceedings of the 6th International Conference on Nuclei Far from Stability and the 9th International Conference on Atomic Masses and Fundamental Constants, Bernkastel-Kues, Germany, 1992, edited by R. Neugart and A. Wöhr (Institute of Physics, Bristol, 1993), p. 193. 